\title{
Complexity, Chaos and Fluctuations
}

\author{
Santo Banerjee ${ }^{1,2, a}$ and Matteo Colangeli ${ }^{3, b}$ \\ 1 Institute for Mathematical Research, University Putra Malaysia, Serdang, Malaysia \\ 2 Malaysia-Italy Centre of Excellence for Mathematical Science, University Putra \\ Malaysia, Serdang, Malaysia \\ 3 Dipartimento di Ingegneria e Scienze dell'Informazione e Matematica, Università degli \\ Studi dell'Aquila, Via Vetoio, 67100 L' Aquila, Italy
}

Received 11 May 2017

Published online 4 July 2017

\begin{abstract}
This EPJ special topics issue is a collection of contributions about some recent developments in statistical mechanics and dynamical complexity. The various articles report results on statistical fluctuations, non equilibrium models, chaos and nonlinear interactions, complexity and its various associated measures.
\end{abstract}

The long-standing endeavor of Statistical Mechanics is to establish a mathematical framework which allows to encompass different levels of description of a many-particle system: (i) the microscopic setting, stemming from the Boltzmann-Gibbs statistical ensemble theory $[1,2]$; (ii) the mesoscopic scale, typically described in the framework of kinetic theory [3-5], interacting particle systems [6-12] or even more general stochastic processes $[13,14]$; (iii) the macroscopic level, which is the standard set-up in contexts such as the derivation of hydrodynamic equations, turbulence [15-17], Irreversible Thermodynamics [18], etc. Equilibrium phenomena have been investigated and understood much more thoroughly than non-equilibrium ones. At present, the equilibrium theory may be considered complete, for what concerns the microscopic foundations of equilibrium thermodynamics, including the theory of phase transitions and critical phenomena. On the other hand, in spite of the long-standing research activity, Nonequilibrium Statistical Mechanics is still an open field of research. One of the reasons is that nonequilibrium phenomena are so numerous, diverse and complex that attaining a unified description may still appear as an elusive ambition. In particular, away from equilibrium one cannot bypass the analysis of the microscopic dynamics even in the study of stationary states, that are the simplest beyond equilibrium.

Nevertheless, relevant progresses were made in this field in the last decades, cf. [19]. We refer, for instance, to the discovery of the Fluctuation Relations for deterministic and stochastic dynamics [20-22]. Much effort was needed, in particular, to identify the minimal mathematical ingredients as well as the physical mechanisms lying beneath the validity of such relations [23-25]. Another result, whose mathematical theory was originally dug out in the sixties of the last century $[26,27]$ and was then gradually

\footnotetext{
a e-mail: santoban@gmail.com

b e-mail: matteo.colangeli1@univaq.it
} 
unravelled in the following decades, is represented by the Fluctuation-Dissipation Theorem [28-33], which allows to express the response of a system to an external perturbation in terms of a correlation function computed with respect to a reference measure.

The different techniques proposed to investigate the variety of non-equilibrium models can be thus considered as a theoretical laboratory in which many fundamental questions in statistical physics can be investigated thoroughly.

Strictly linked to the framework of Nonequilibrium Statistical Mechanics is the emerging, rather interdisciplinary, realm of "Complexity". Complexity is a term associated with a complex or complex adaptive system, which measures the amount of information related to the dynamics. Complex system is one, which has many interacting components that make the whole unit highly nonlinear. Complexity measures are of trending interest in both classical and quantum systems. It is very effective to quantify the 'uncertain' phenomenon in several branches of Science, Engineering and Management [34-39]. For example, the structure of an organization is complex, may not be chaotic. The healthy human heart is complex, since it has many interacting subunits to keep the whole system (heart) active. A cardiac heart is less complex, since it fails to working properly with each interacting subunits [40,41]. Several other type of complexity can be investigated in laser and optics [34], ecological and biological models and networks [40,41], pseudo random number generations [35], economy and politics [39], fractional dynamics [42], etc.

Dynamical complexity is generally investigated by computing the entropy of the dynamics. In fact the Shannon entropy and the von Neumann entropy are related to the information present in classical and quantum systems, respectively. Entropy, in information theory point of view, measures the amount of uncertainty in a dynamics. It increases when the system is more likely random and decreases, when it is less random. Thus, if the entropy is high for some system, the system is considered more complex, while for low entropy value, the system is less complex. Several entropy measures such as Shannon entropy, Gibbs entropy, Kolmogorov-Sinai entropy, have got tremendous response in the research communities. Since the inception of the recurrence plots $(\mathrm{RP})$, few other measures of complexity have been introduced, which stands more effective than the Lyapunov exponent in determining the divergence behavior of dynamical systems. Such entropy based quantifiers of RPs includes normalized entropy of recurrence times or the Shannon entropy of the distribution of length of diagonal line segments, which can detect points of bifurcation. However, these RP based entropy, sometimes fail to capture the complexity of the system properly because of the choice of improper distance threshold to obtain the binary recurrent matrix. Thus, an alternative notion of Weighted RP(WRP) was introduced. The Shannon entropy based on WRP is known as Entropy of the WRP (WRPE).

This special issue on 'Aspects of Statistical Mechanics and Dynamical Complexity', consists of 15 regular research articles and one review note. Each contribution refers to the trends of statistical mechanics, the recent developments on complexity and complex networks. Several analytical and experimental results are combined together. The articles can be grouped into six categories, namely:

1. Complexity of systems, networks and signals [36,43-46];

2. Complexity and random number generators $[35,47]$;

3. Fluctuations and stochastic dynamics [48,49];

4. Coupled transport problem and maximum entropy principle [50,51];

5. Quantum mechanics in phase space [52];

6. Modeling and various applications [53-56] .

In [43], the authors investigated the dynamical complexity by Shannon entropy is not an effective measure of short and noisy time series from chaotic dynamical 
systems and Markov chains. They proposed a new measure for short and noisy time series. The analysis has been implemented with analytical and simulation results.

The article [44] is about connections between hierarchical topological scales of the simplicial complexes and the organization of functional clusters communities in complex networks. The analysis has been implemented analytically and also with random network configurations with large number of nodes.

In [36] the authors proposed the changes in the dynamics and complexity in synchronized and non synchronized states. They also proposed and modified a chaos synchronization based secure communication scheme to implement in case of non synchronization. The scheme is designed and illustrated using examples and simulations.

In [45] the authors investigated the high dynamical complexity of the brain, as a small world topology. They compared four nonlinear connectivity measures and show that each method characterizes distinct features of brain interactions.

The paper by Cerbelli et al. [46] studies the properties of laminar mixing flows exploiting chaotic advection and investigate the qualitative and quantitative behavior of the eigenvalues/eigenfunctions of the advection-diffusion operator in the limit of large Peclet numbers. In particular, the use of the mapping-matrix approach, calibrated to account for coarse-graining effects, is used by the Authors to investigate a wide range of Peclet values.

The article [47] is about a novel method to extend the Cat map from 2-dimension to higher dimension using the fast pseudo Hadamard Transform. The associated complexity is investigated using various entropy measures.

In [35] the authors proposed a topologically conjugate map, equivalent to the Logistic map. The proposed map is on integer domain, which is effective for classical cryptography. Permutation entropy (PE) and the Lempel-Ziv (LZ-76) complexity measure has been implemented for both the maps to compare the dynamical properties.

The research by Giona et al. [48] analyzes the thermodynamic properties of stochastic differential equations driven by smooth Poisson-Kac fluctuations, and their convergence, in the Kac limit, towards Wiener-driven Langevin equations. In particular, using a Markovian embedding of the stochastic work variable, the Authors prove that the Kac-limit convergence implies a Stratonovich formulation of the limit Langevin equations.

The article by Boccagna et al. [49] explains in detail how to construct an efficient algorithm based on the Multispin Coding technique for the Kob-Andersen glassy dynamics. It is worth recalling that when ergodicity is broken, computer simulations are essential to keep track of dynamical aspects. However, since the dynamics is inherently slow, execution times may be really long; here comes, hence, the need to speed up simulations by using fast algorithms to provide outputs in suitable times.

The article by Auletta et al. [50] tackles a fundamental issue in Statistical Mechanics, namely the validity of the so-called Maximum Entropy Principle away from equilibrium. After a careful examination of some paradigmatic examples, the Authors are prone to conclude that the above principle does not actually have a predictive nature and can yield wrong results for nonequilibrium systems. In particular, the conclusion of the Authors, shared also by us, is that the analysis of the microscopic dynamics is a crucial step not to be avoided when dealing with systems away from equilibrium.

The article by Duong et al. [51] explores the realm of hydrodynamic limits for interacting particle systems. In particular, the Authors investigate which properties of the microscopic dynamics must be invoked to give rise to a particular structure of macroscopic coupled transport equations arising when different populations are jointly evolving. This manuscript, hence, fits definitely well with the scope of the 
Special Issue in that it sheds light on the bridge between different levels of description of a particle system.

In [52] the authors constructed the Wigner functions of non commutative quantum mechanics for a system of 2-degrees of freedom. Wigner functions are known to be the quantum-mechanical analogue of the phase space distribution of classical statistical mechanics that includes quantum mechanical corrections and hence serve many computational purposes in quantum settings. It is also known that the Wigner function can be related to variation of information measures and entropies.

The article [53] is a review work on the security of multi threaded program.The authors discussed about existing and a novel model which does not follow the traditional information-theoretic channel setting. The quantitative analysis is discussed with entropy measures.

The article [54] is about experimental observation of Fuzzy-PD Controller for a moving robot. The analysis has been investigated with a Cross-Entropy Optimization Approach.

The paper by Rodrìguez et al. [55] proposes a suitable variation of the "Infotaxis" mechanism, which is an olfactory search strategy recently proposed in the literature to locate the source of a volatile substance transported in a turbulent environment. The Authors consider a slightly modified strategy called blind-infotaxis and study its performance measured by the rate of success and mean search time, under changes in the environment parameters such as diffusivity, rate of emission or wind velocity. Their results pose some limits on the performance of blind Infotaxis, and also have practical consequences for the design of future machines able to track and detect an emitting source of chemicals or volatile substances.

The paper by Cirillo et al. [56] deals, instead, with the issue of Metastability which is a phenomenon widely studied both in the mathematical and physical literature. The Authors, under rather general conditions, give an estimate of the exit time from a metastable state in a presence of a second metastable state that must be necessarily visited by the system before eventually reaching the stable phase.

We would like to thank the authors for their contributions and the referees for their remarkable effort in reviewing the articles. We hope this issue will be helpful for the scientists and researchers working on the field of statistical mechanics. Finally, we would like to thank all members of EPJ ST, for hosting this special issue, specially Dr. Caron, Sabine Lehr and Nicolas Puyaubreau for their kind support.

\section{References}

1. G. Gallavotti, Statistical Mechanics. A Short Treatise (Springer-Verlag, Berlin Heidelberg, 1999)

2. J.R. Dorfman, An Introduction to Chaos in Nonequilibrium Statistical Mechanics (Cambridge University Press, 1999)

3. I.V. Karlin, M. Colangeli, M. Kröger, Exact linear hydrodynamics from the Boltzmann equation, Phys. Rev. Lett. 100, 214503 (2008)

4. M. Colangeli, M. Kröger, H.C. Öttinger, Boltzmann equation and hydrodynamic fluctuations, Phys. Rev. E 80, 051202 (2009)

5. M. Colangeli, From Kinetic Models to Hydrodynamics. Some Novel Results. SpringerBriefs in Mathematics (Springer, New York, 2013)

6. C. Kipnis, C. Landim, Scaling Limits of Interacting Particle Systems (Springer-Verlag, Berlin, Heidelberg, 1999)

7. E. Cirillo, M. Colangeli, A. Muntean, Does communication enhance pedestrians transport in the dark? C. R. Mecanique 344, 19 (2016) 
8. E. Cirillo, M. Colangeli, A. Muntean, Stationary currents in particle systems with constrained hopping rates, J. Non-Equilib. Thermodyn. 41, 99 (2016)

9. E. Cirillo, M. Colangeli, A. Muntean, Effects of communication efficiency and exit capacity on fundamental diagrams for pedestrian motion in an obscure tunnel - a particle system approach, Multiscale Model. Simul. 14, 906 (2016)

10. E. Cirillo, M. Colangeli, A. Muntean, Blockage-induced condensation controlled by a local reaction, Phys. Rev. E 94, 042116 (2016)

11. M. Colangeli, A. De Masi, E. Presutti, Latent heat and the Fourier law, Phys. Lett. A 380, 1710 (2016)

12. M. Colangeli, A. De Masi, E. Presutti, Particle models with self-sustained current, J. Stat. Phys. 167, 1081 (2017)

13. M. Baiesi, C. Maes, B. Wynants, Nonequilibrium linear response for Markov dynamics, I: jump processes and overdamped diffusions, J. Stat. Phys. 137, 1094 (2009)

14. M. Colangeli, C. Maes, B. Wynants, A meaningful expansion around detailed balance, J. Phys. A: Math. Theor. 44, 095001 (2011)

15. M. Colangeli, I.V. Karlin, M. Kröger, From hyperbolic regularization to exact hydrodynamics for linearized Grad's equations, Phys. Rev. E 75, 051204 (2007)

16. M. Colangeli, I.V. Karlin, M. Kröger, Hyperbolicity of exact hydrodynamics for threedimensional linearized Grad's equations, Phys. Rev. E 76, 022201 (2007)

17. T. Bohr, M.H. Jensen, G. Paladin, A. Vulpiani, Dynamical Systems Approach to Turbulence (Cambridge University Press, 1998)

18. H.C. Öttinger, Beyond Equilibrium Thermodynamics (Wiley \& Sons, 2005)

19. M. Colangeli, L. Rondoni, A. Verderosa, Focus on some nonequilibrium issues, Chaos Solitons Fractals 64, 2 (2014)

20. G. Gallavotti, E.G.D. Cohen, Dynamical ensembles in nonequilibrium statistical mechanics, Phys. Rev. Lett. 94, 2694 (1995)

21. D.J. Evans, D.J. Searles, The fluctuation theorem, Adv. Phys. 52, 1529 (2002)

22. J.L. Lebowitz, H. Spohn, A Gallavotti-Cohen-type symmetry in the large deviation functional for stochastic dynamics, J. Stat. Phys. 95, 333 (1999)

23. M. Colangeli, R. Klages, P. De Gregorio, L. Rondoni, Steady state fluctuation relations and time-reversibility for non-smooth chaotic maps, J. Stat. Mech.: Theor. Exp. 2011, P04021 (2011)

24. M. Colangeli, L. Rondoni, Equilibrium, fluctuation relations and transport for irreversible deterministic dynamics, Physica D 241, 681 (2012)

25. P.A. Adamo, M. Colangeli, L. Rondoni, Role of ergodicity in the transient Fluctuation Relation and a new relation for a dissipative non-chaotic map, Chaos Solitons Fractals 83, $54(2016)$

26. R. Kubo, Statistical-mechanical theory of irreversible processes: I. General theory and simple applications to magnetic and conduction problems, J. Phys. Soc. Japan 12, 570 (1957)

27. R. Zwanzig, Nonequilibrium Statistical Mechanics (Oxford University Press, 2001)

28. D. Ruelle, General linear response formula in statistical mechanics, and the fluctuationdissipation theorem far from equilibrium, Phys. Lett. A 245220 (1998)

29. B. Marini, U. Marconi, A. Puglisi, L. Rondoni, A. Vulpiani, Fluctuation-dissipation: response theory in statistical physics, Phys. Rep. 461, 111 (2008)

30. B. Cessac, J.-A. Sepulchre, Linear response, susceptibility and resonances in chaotic toy models, Physica D 225, 13 (2007)

31. M. Colangeli, L. Rondoni, A. Vulpiani, Fluctuation-dissipation relation for chaotic nonHamiltonian systems, J. Stat. Mech.: Theor. Exp. 2012, L04002 (2012)

32. V. Lucarini, M. Colangeli, Beyond the linear fluctuation-dissipation theorem: the role of causality, J. Stat. Mech.: Theor. Exp. 2012, P05013 (2012)

33. M. Colangeli, V. Lucarini, Elements of a unified framework for response formulae, J. Stat. Mech.: Theor. Exp. 2014, P01002 (2014)

34. L. Rondoni, M.R.K. Ariffin, R. Varatharajoo, S. Mukherjee et al., Opt. Commun. 387, $257(2017)$

35. M.R.M. Said, A.D. Hina, S. Banerjee, Eur. Phys. J. Special Topics 226, 2281 (2017) 
36. S.K. Palit, N.A.A. Fataf, M.R.M. Said, S. Mukherjee, S. Banerjee, Eur. Phys. J. Special Topics 226, 2219 (2017)

37. S. Banerjee, J. Kurths, E. Scholl, Eur. Phys. J. Special Topics 225, 1 (2016)

38. T.S. Dang, S.K. Palit, S. Mukherjee, T.M. Hoang, S. Banerjee, Eur. Phys. J. Special Topics 225, 159 (2016)

39. S.S. Ercetin, S. Banerjee, Chaos, Complexity and Leadership, Chaos, Complexity and Leadership 2013 (Springer, 2015)

40. S. Banerjee, S.K. Palit, S. Mukherjee, M.R.K. Ariffin, L. Rondoni, Chaos 26, 033105 (2016)

41. S. Mukherjee, S.K. Palit, S. Banerjee, M.R.K. Ariffin, L. Rondoni et al., Physica A 439, $93(2015)$

42. S. He, K. Sun, S. Banerjee, Eur. Phys. J. Plus 131, 254 (2016)

43. N. Nagaraj, K. Balasubramanian, Eur. Phys. J. Special Topics 226, 2191 (2017)

44. V. Berec, Eur. Phys. J. Special Topics 226, 2205 (2017)

45. L.M. Uchoa da Silva, C. Arruda Baltazar, C. Aquemi Silva, M. Watanabe Ribeiro, M.A. Albano de Aratanha, C. Sardeto Deolindo, A. Cardoso Rodrigues, B. Soares Machado, Eur. Phys. J. Special Topics 226, 2235 (2017)

46. S. Cerbelli, M. Giona, O. Gorodetskyi, P.D. Anderson, Eur. Phys. J. Special Topics 226, $2247(2017)$

47. T.T.K. Hue, T.M. Hoang, Eur. Phys. J. Special Topics 226, 2263 (2017)

48. M. Giona, A. Brasiello, S. Crescitelli, Eur. Phys. J. Special Topics 226, 2299 (2017)

49. R. Boccagna, Eur. Phys. J. Special Topics 226, 2311 (2017)

50. G. Auletta, L. Rondoni, A. Vulpiani, Eur. Phys. J. Special Topics 226, 2327 (2017)

51. M.H. Duong, A. Muntean, O.M. Richardson, Eur. Phys. J. Special Topics 226, 2345 (2017)

52. S. Hasibul Hassan Chowdhury, H. Zainuddin, Eur. Phys. J. Special Topics 226, 2359 (2017)

53. T.M. Ngo, M. Huisman, Eur. Phys. J. Special Topics 226, 2375 (2017)

54. D.N. Anisimov, T.S. Dang, S. Banerjee, T.A. Mai, Eur. Phys. J. Special Topics 226, $2393(2017)$

55. J. Duque Rodriguez, D. Gomez-Ullate, C. Mejia-Monasterio, Eur. Phys. J. Special Topics 226, 2407 (2017)

56. E.N.M. Cirillo, F.R. Nardi, C. Spitoni, Eur. Phys. J. Special Topics 226, 2421 (2017) 